\title{
A New Life Prediction Scheme for Mechanical System with Considering the Mission Profile Switching
}

\author{
Jiancheng Yin $₫$, Huailiang Zheng, Yuantao Yang $®$ and Minqiang $\mathrm{Xu}$ * \\ Deep Space Exploration Research Center, Harbin Institute of Technology, Harbin 150001, China; \\ wdyydy@163.com (J.Y.); hlzhenghit@126.com (H.Z.); yangyuantaohit@163.com (Y.Y.) \\ * Correspondence: xumqhit15@126.com
}

Received: 1 December 2019; Accepted: 15 January 2020; Published: 18 January 2020

\begin{abstract}
The life prediction is crucial to guarantee the reliability and safety of the mechanical system. The current prediction methods predict the life only based on the historical usage pattern of the mechanical system, and do not consider the mission profile of the future working process. To realize the life prediction with considering the switching of mission profile, which is composed of different operating conditions, this paper proposes a new prediction scheme on the base of the similarity trajectory method (STM). Two main improvements are employed. First, the reference degradation models are constructed according to the predicted trend of each constant operating condition obtained by the relevance vector machine (RVM). Secondly, the life under specific mission profile is calculated through weighted aggregating the life of each constant operating condition. The proposed method is validated by a turbofan engine simulation data. The results show that the proposed method achieves an excellent predicted result, in which the predicted result is close to the actual result. In addition, the proposed method can deal with the problem of mission profile switching.
\end{abstract}

Keywords: mission profile; operating conditions; relevance vector machine; life prediction; similar trajectory

\section{Introduction}

Prognostics and health management (PHM) is very crucial in modern industries, as the mechanical system is becoming larger, more complicated, and more automated. Traditional maintenance strategies such as breakdown corrective maintenance and scheduled preventive maintenance find it hard to deal with the increasing demand of reliability and efficiency. Prognostic, which is one part of the PHM system, has been widely researched in recent years [1,2]. It is a key technique to predict the remaining useful life (RUL) of the machine and further to establish reasonable maintenance plans. Therefore, the unplanned downtime, the maintenance cost, and the safety risk can be reduced.

The life prediction methods can be roughly divided into two categories: Model-based methods and data-driven methods. Although the model-based methods [3-5] have clear theoretical basis and high prediction accuracy, it is still very difficult to establish and solve the physical model of complicated equipment. With the advantage that need not construct a complicated physical model, data-driven methods have been extensively studied in the past decades. For example, a recurrent neural network based the health indicator of bearing was proposed by Guo et al. [6]. Zhu et al. [7] estimated the RUL of bearing by using the time-frequency features and multi-scale convolutional neural network. Ren et al. [8] proposed a new deep learning method-based prediction framework for bearing RUL prediction by using an auto-encoder and deep neural networks (DNNs). As the long short-term memory (LSTM) neural network can be used as a complex non-linear element to construct a larger depth neural network, the LSTM neural network $[9,10]$ is also used to predict the RUL. The abovementioned methods can all achieve superior prediction results under a single operating condition. However, the change of 
different operating condition is the more general scenario in actual applications. For the situation of variable operation conditions, Wu et al. [11], Jiang et al. [12], Rigamonti et al. [13], and Yan et al. [14] only predicted the RUL under variable operating conditions by putting forward innovative characteristic parameters or health indicators, but did not propose the prediction method suitable for variable operating conditions. Although Sameer et al. [15] and Tao et al. [16] proposed the prediction methods suitable for the variable operating condition by using the historical data and Monte Carlo simulation, the complexity of the calculation process limited the wide use of the methods. Furthermore, the similarity trajectory method (STM) [17-21], which is simpler to calculate, was widely used to predict the RUL of the mechanical system under variable operating conditions [22-26]. The STM is an effective method to deal with the life prediction under variable operating conditions.

However, the precondition of all the methods mentioned above is that the operating condition in the prediction process should be consistent with that of the training samples. This means that the life of mechanical systems is an extrapolation of the current state. Nevertheless, in order to accomplish different tasks, the mechanical systems often need to constantly switch mission profile (detailed definition and description is give in Section 2) in practice. For the mission profile switching in the prediction, the Bayesian technique [27], Markov process [28], and Wiener process [29] are widely used to deal with the problem. Besides, Gebraeel et al. [30] proposed a stochastic mathematical model to calculate the residual life distributions of partially degraded systems. Zhao et al. [31] proposed an integrated prognostics method for gear under time-varying operating conditions with considering the influence of uncertainty. Zhang et al. [32] proposed a prognostic model for the system with considering the performance degrades with usage and recovers in storage.

Although the above method can consider the switching problem of mission profile in the prediction process, the switched mission profile is composed of constant operating conditions. The above method cannot yet solve the problem in which the mission profile is composed of variable operating conditions. But the mission profile with variable operating conditions is the most frequently encountered state of mechanical systems.

Motivated by the abovementioned problems, this paper proposes a new life prediction scheme for the mechanical system with considering the mission profile switching in the prediction process. First, the prediction trend and the lifecycle of each constant operating condition of the similarity reference samples are used to construct the reference degradation models. Then, the predicted life of each operating condition of the sample to be predicted is obtained according to STM. Finally, according to the proportion of each operating condition in the specific mission profile, the life of the sample to be predicted under specific mission profile is calculated through weighted aggregating the life of each constant operating condition of the sample to be predicted. In order to solve the switching of the mission profile with variable operating conditions, in the method, two main improvements on the base of the STM are proposed:

(1) For the lifecycle degradation curves collected under variable operating conditions (several single operating conditions are included) in the training dataset, predict each life-cycle degradation curve under constant operating conditions (for each single operating condition, predict a degradation curve) using the relevance vector machine (RVM).

(2) Calculate the proportions of each single operating condition in the mission profile, then predict the life of the samples to be predicted through weighted aggregating the life of the constant operating conditions.

The rest of this paper is organized as follows. Section 2 describes the problem to be addressed in detail. In Section 3, the proposed method is introduced in detail. The process and analysis of the proposed method are explained in Section 4 to verify the effectiveness of the proposed method. Finally, the conclusion is drawn in Section 5. 


\section{The Description of the Problem}

In this section, the problems to be solved by the proposed method will be further elaborated, and the definition of the mission profile should be given before the explanation. The definition of the mission profile is given as follows.

Mission profile [33]: A time-phased description of the events and environments experienced by an item during a given mission. The profile depicts the time spans of the events and operational conditions to be anticipated. In order to illustrate more clearly, in the following sections, the mission profile is simply defined as a time-phased description of the operating condition by an item during a given mission.

As shown in Figure 1, the mission profile is constituted by different operating conditions in a certain order. Meanwhile, the life trend and lifecycle of the mechanical system are affected by the mission profile. In the actual production process, in order to effectively complete different production tasks, the mission profile will be switched accordingly. Therefore, the life trend of the mechanical system will also change accordingly. In addition, each mission profile also consists of more than one operating condition.

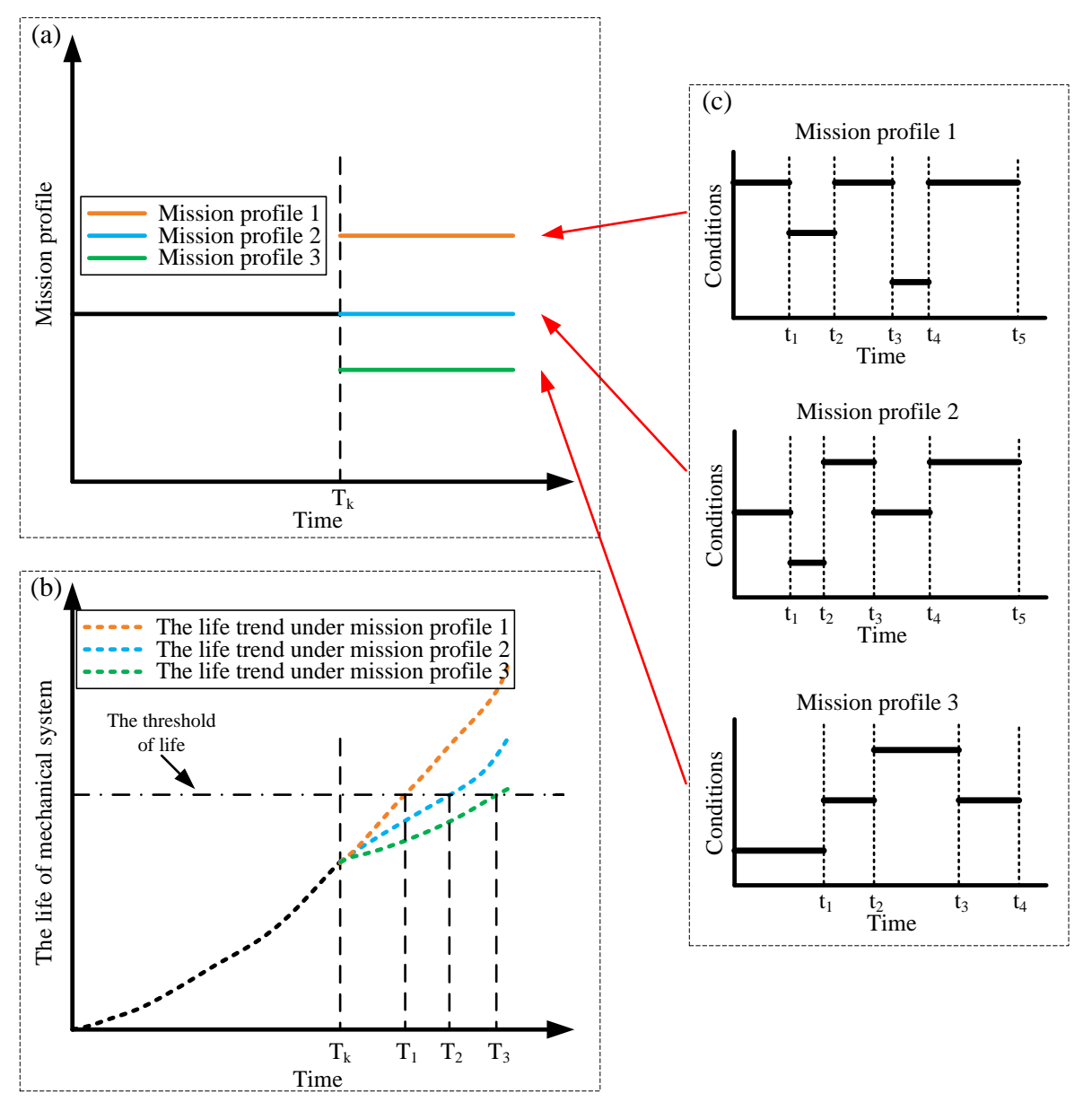

Figure 1. The relationship among operating condition, mission profile, and lifecycle. (a) The mission profile experienced by the mechanical system. (b) The life trend of the mechanical system under different mission profiles. (c) The composition of the mission profile. The mission profile is constituted by operating condition in a certain order as shown in $(\mathbf{c}-\mathbf{a})$. The life trend and lifecycle are affected by the mission profile as shown in $(\mathbf{a}, \mathbf{b})$. $T_{k}$ is the switch point of mission profile. $T_{1}, T_{2}$, and $T_{3}$ is the lifecycle under the mission profiles 1,2 , and 3 , respectively. $t_{1}, t_{2}, t_{3}, t_{4}$, and $t_{5}$ is the switch point of different operating condition. 
However, most of the existing prediction methods are based on the premise that the mission profile before and after prediction are identical. Only one constant mission profile cannot solve the actual prediction of the mechanical system, as the actual mechanical system cannot always work under a constant mission profile without switching. Although some prediction methods considered the switching of operating conditions, each switching is only a constant operating condition rather than the mission profile switching under different operating conditions. Therefore, the existing prediction methods cannot effectively solve the life prediction of the actual mechanical system under the mission profile, which consists of different operating condition switching.

Therefore, in this paper, a method to solve the life prediction of the actual mechanical system under mission profile switching is proposed. That is to say, the mission profile at the time of prediction does not necessarily coincide with the known mission profile. Meanwhile, each mission profile consists of different operating conditions. The proposed method is described below.

\section{The Proposed Method}

According to the above description, the mission profile of the mechanical system will seriously affect its service life. Therefore, in the process of life prediction, the accuracy of life prediction can be improved by considering a specific mission profile in the future. In order to achieve the life prediction with considering the future mission profile, the main implementation steps of the proposed method are as follows.

Step 1: Select several similarity reference samples from the historical samples through measuring the similarity between the samples to be predicted and all the samples in the historical sample.

Step 2: Operating condition partitioning. Given a similar reference sample sequence $S T^{i}=$ $\left\{x_{1}, \ldots, x_{m}\right\}$, partition this sequence into $C$ new sequences $\left\{O T_{j}^{i} j j=1, \ldots, C\right\}$ according to the operating condition, where $\boldsymbol{O} T_{j^{\prime}}^{i} \cap_{j \neq j^{\prime}} \boldsymbol{O} \boldsymbol{T}_{j}^{i}=\varnothing$. Where $m$ is the number of similar reference samples and $C$ is the number of operating conditions.

Step 3: Construct the reference degradation model by using RVM.

Step 4: Calculate the proportions of each single operating condition in the future mission profile, then predict the life through weighted aggregation.

The basic framework of the proposed method is shown in Figure 2. The proposed method has two critical steps: The construction of the reference degradation model and the life prediction according to the future mission profile. The two steps are introduced in detail as follows.

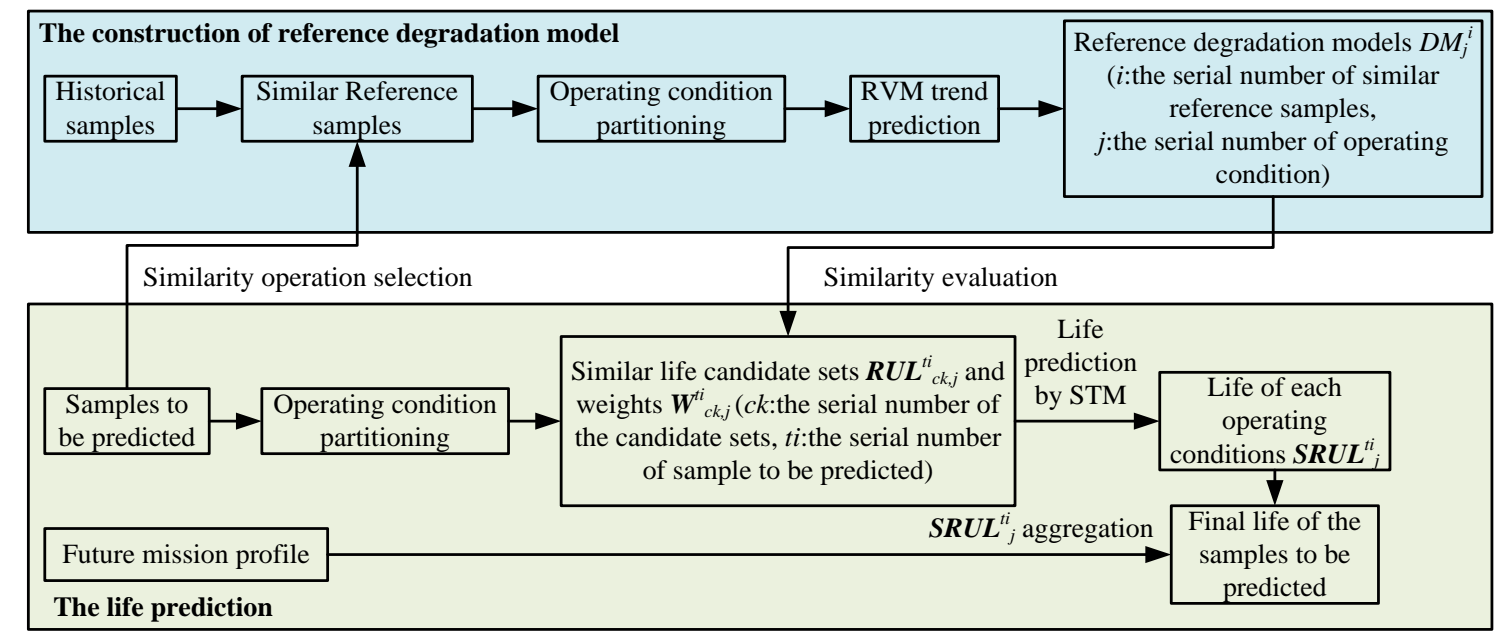

Figure 2. The process of the proposed method. The proposed method consists of two main processes: The construction of the reference degradation model and the life prediction. RVM, relevance vector machine. 


\subsection{The Construction of the Reference Degradation Model}

In the proposed method, the reference degradation models are constructed based on the prediction results of each constant operating condition of the reference samples instead of the historical lifecycle data of the reference samples. By this way, not only can the final life prediction results be more accurate, but also the life prediction under different mission profiles can be achieved. After obtaining the partitioned operating conditions $\boldsymbol{O T _ { j }}{ }^{i}$ of similar reference samples by Step 2, the reference degradation models $D M_{j}{ }^{i}$ can be built as follows. Where $i=1,2, \ldots, m, j=1,2, \ldots, C, m$ is the number of similar reference samples, and $C$ is the number of operating conditions.

Step 3.1: Calculate the standardized samples. The standardized samples $N T_{j}{ }^{i}$ of the partitioned operating conditions are calculated as follows.

$$
\boldsymbol{N} \boldsymbol{T}_{j}{ }^{i}=\frac{\boldsymbol{O} \boldsymbol{T}_{j}{ }^{i}-\operatorname{mean}\left(\boldsymbol{O} \boldsymbol{T}_{j}{ }^{i}\right)}{\operatorname{std}\left(\boldsymbol{O} \boldsymbol{T}_{j}{ }^{i}\right)}
$$

Step 3.2: Extract the maximum value of the standardized samples. The maximum value of the standardized samples is used as the life terminal point of each constant operating condition in one reference sample and is obtained as follows.

$$
N M^{i}=\max _{0 \leq j \leq C}\left(\max \left(N T_{j}^{i}\right)\right)
$$

where, for the standardized sample, the maximum value only needed to be calculated once. However, for the standardized samples $N T_{j}{ }^{i}$ of the partitioned operating conditions, the maximum value of each partitioned operating condition should be calculated first, then the maximum of the maximum value of each partitioned operating condition is the maximum value of the standardized samples.

Step 3.3: Calculate the life thresholds of each constant operating condition in one reference sample. According to the maximum value of the standardized samples and the partitioned operating conditions, the life threshold $O M_{j}{ }^{i}$ of each constant operating condition can be obtained.

$$
O M_{j}^{i}=N M^{i} \cdot \operatorname{std}\left(O T_{j}^{i}\right)+\operatorname{mean}\left(\boldsymbol{O} \boldsymbol{T}_{j}^{i}\right)
$$

As in Step 4.2, we should calculate the life of each operating condition of the sample to be predicted according to STM. Therefore, it is necessary to calculate the similarity between each operating condition of the sample to be predicted and that of similar reference samples. However, according to Equation (1), if we want to calculate the standardized sample of each operating condition, we must know the mean and variance of the operating condition. For the similar reference samples, each operating condition is the data under the whole life. So the mean and variance of each operating condition can be easily obtained. However, for the sample to be predicted, each operating condition is not the data under the whole life. Therefore, it is impossible to standardize the operating condition of the sample to be predicted reasonably and effectively. Thus, it is necessary to restore the endpoint of similar reference samples to values before standardization according to Equation (3).

Step 3.4: Predict the lifecycle degradation curve of each constant operating condition. According to the life thresholds of each constant operating condition, the lifecycle degradation curve of each constant operating condition is predicted by RVM, respectively. The specific process of RVM refers to $[34,35]$.

Step 3.5: Build the reference degradation models. According to the prediction results of the lifecycle degradation curve in Step 3.4, the trend $\boldsymbol{R} \boldsymbol{T}_{j}{ }^{i}$ and the lifecycle $L T_{j}{ }^{i}$ of each constant operating condition are used to construct the reference degradation models $D M_{j}{ }^{i}$.

Figure 3 is the process of the reference degradation models construction. 


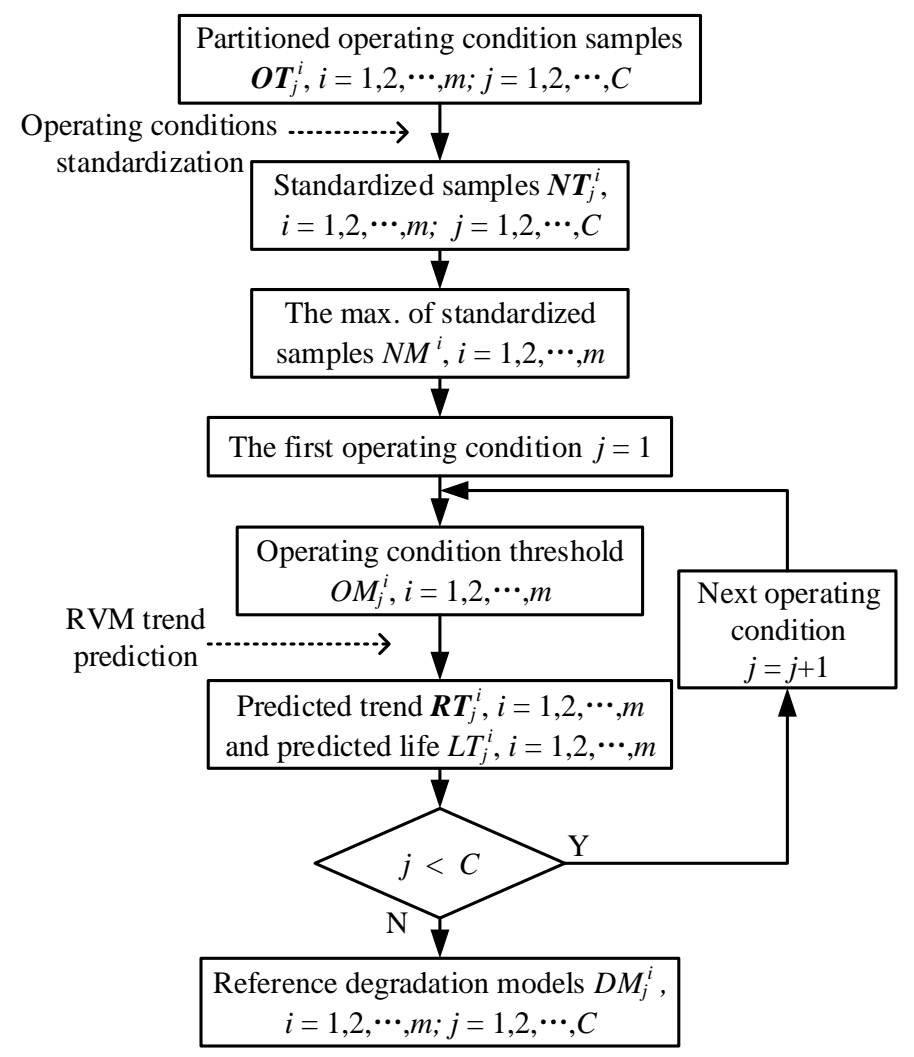

Figure 3. The process of the reference degradation models constructing.

\subsection{The Life Prediction According to Specific Task Requirement}

The general procedure of the final life prediction with considering the mission profile is illustrated as Figure 4. According to the reference degradation models, the final life of the samples to be predicted under specific mission profile can be obtained as follows.

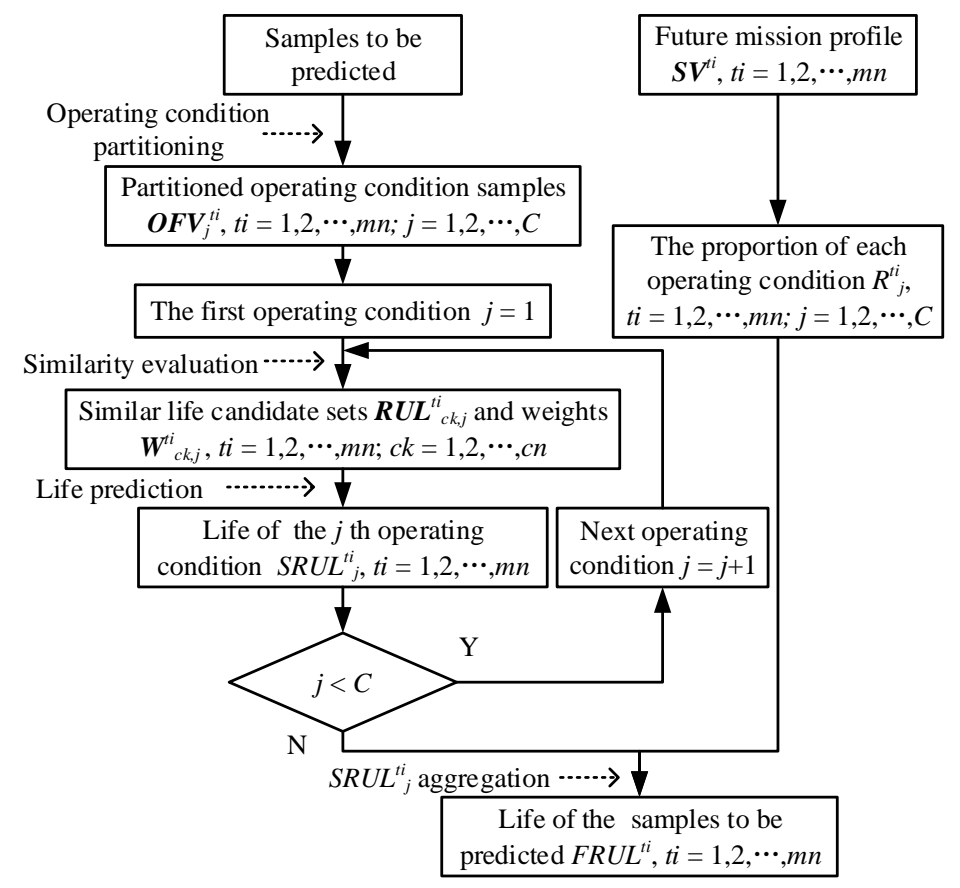

Figure 4. The process of the life prediction. 
Step 4.1: Partition the operating condition of the samples to be predicted. For the samples to be predicted, partition this sequence into $C$ new sequences $\left\{O F V_{j}^{t i} \mid j=1, \ldots, C\right\}$ according to the

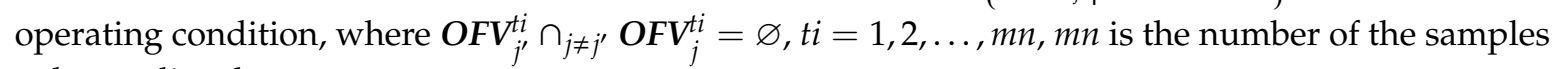
to be predicted.

Step 4.2: Predict the life of each constant operating condition of the test samples. According to the STM, in which the specific process refers to [20,21], the life candidate sets $\boldsymbol{R} U \boldsymbol{L}_{c k, j}^{t i}$ and the weights $W_{c k, j}^{t i}$ can be obtained, where $c k=1,2, \ldots, c n, c n$ is the number of the candidate sets. Then, the life of each constant operating condition $S R U L_{j}^{t i}$ of the samples to be predicted can be calculated as follows.

$$
S R U L_{j}^{t i}=\sum_{c k=1}^{c n} R U L_{c k, j}^{t i} \cdot W_{c k, j}^{t i}
$$

Step 4.3: Obtain the proportion of each operating condition in the mission profile. According to the future mission profile $S V^{t i}$, determine the proportion of each operating condition $R_{j}{ }^{t i}$ which is needed to complete the specific task.

Step 4.4: Predict the final life of the samples to be predicted under a specific mission profile. According to the life of each constant operating condition and the proportion of each constant operating condition in the mission profile, the life of the samples to be predicted $F R U L^{t i}$ under a specific mission profile can be calculated as follows.

$$
F R U L^{t i}=\sum_{j=1}^{C} S R U L_{j}^{t i} \cdot R_{j}^{t i}
$$

\section{Results and Discussion}

The proposed method is validated by the turbofan engine degradation simulation dataset, which is available from the NASA Ames Prognostics Data Repository [36].

\subsection{Data Description}

The turbofan engine degradation model was built by Saxena et al. using Commercial Modular Aero-Propulsion System Simulation (CMAPSS) [37]. The simulation data can accurately show the degradation characteristic of the turbofan engine under various failure modes. Each simulation sample, which consists of 24-dimensional time series ( 3 operating parameters and 21 sensor measurements), provides a whole fault evolution recoding of the turbofan engine throughout its lifecycle. The detailed information of 21-dimensional parameters refers to [37], where the 3 operating parameters for turbofan engine can be divided into 6 operating conditions, as shown in Figure 5a. Where three axes respectively denote three operating parameters, each point in the figure denotes one specific operating condition decided by three different parameters.

The monitoring signal of channel 3 is shown in Figure $5 \mathrm{~b}$. The obvious degradation trend cannot be observed directly due to the effect of the random changes in operating condition. In order to clearly observe the degradation trend, the standardized data of the operating condition [38] is shown in Figure 5c. As shown in Figure 5, the simulation data have an obvious degradation trend and contain different operating conditions. Therefore, the simulation data can be used to verify the effectiveness of the proposed method. 
(a)

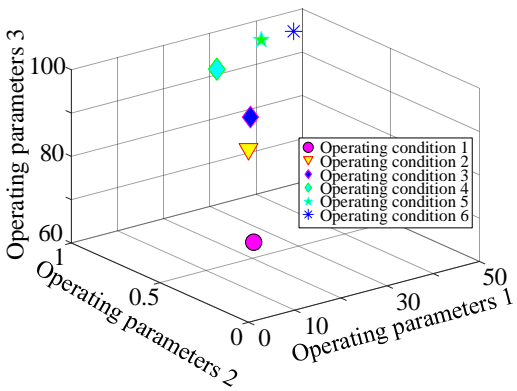

(b)

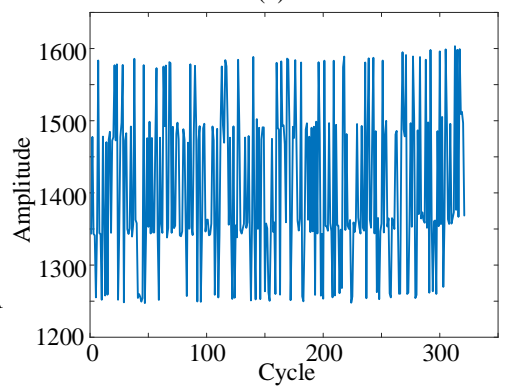

(c)

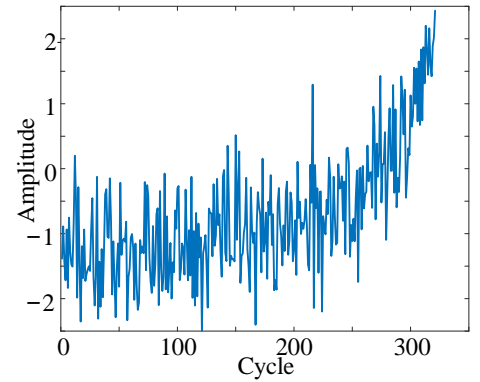

Figure 5. The information of the simulation dataset. (a) The partition of different operating condition. Three axes respectively denote three operating parameters, and each point in the figure denotes one specific operating condition decided by three different parameters. (b) The measured value of the sensor. (c) The standardization of measured value.

\subsection{The Life Prediction Under Mission Profile Switching}

In order to show the calculation process of the proposed method more clearly, lifecycle data in set \#2 of the turbofan engine simulation data from NASA repository are selected to be displayed as a the sample to be predicted. The selected life-cycle data are shown in Figure 6a and the life of the data is 340 cycle. The selected life-cycle data are divided into two parts: The first $2 / 3$ is used as the sample to be predicted, as shown in Figure $6 b$, and the latter $1 / 3$ is used to validate the prediction result under the mission profile experienced by the latter $1 / 3$ data, as shown in Figure $6 c$. The remaining samples in set \#2 are used as the historical sample. Then, the results of the two main steps of the proposed method are shown below.
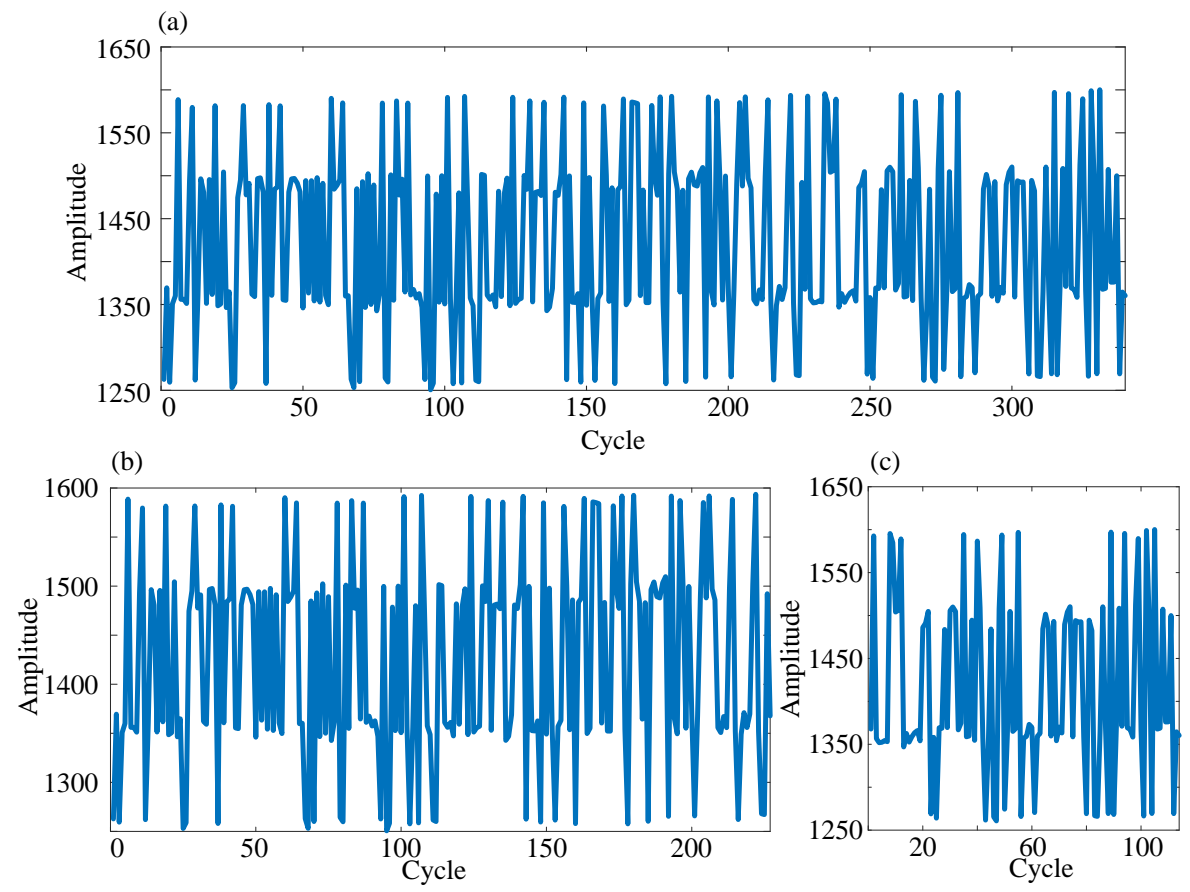

Figure 6. The information of the selected life-cycle data. (a) The selected life-cycle data in set \#2 of the turbofan engine simulation data. (b) The first $2 / 3$ of the selected life-cycle used as the sample to be predicted. (c) The latter $1 / 3$ of the selected life-cycle used to validate the result.

\subsubsection{The Construction of Reference Degradation Model}

For the convenience of explanation, according to Step 1 in Section 3, select three similarity reference samples from the historical samples through measuring the similarity between the samples to be 
predicted, which is shown in Figure $6 \mathrm{~b}$, and the historical sample. One of the similarity reference samples (as shown in Figure 7a) is used as an example to illustrate the construction process of the reference degradation model.
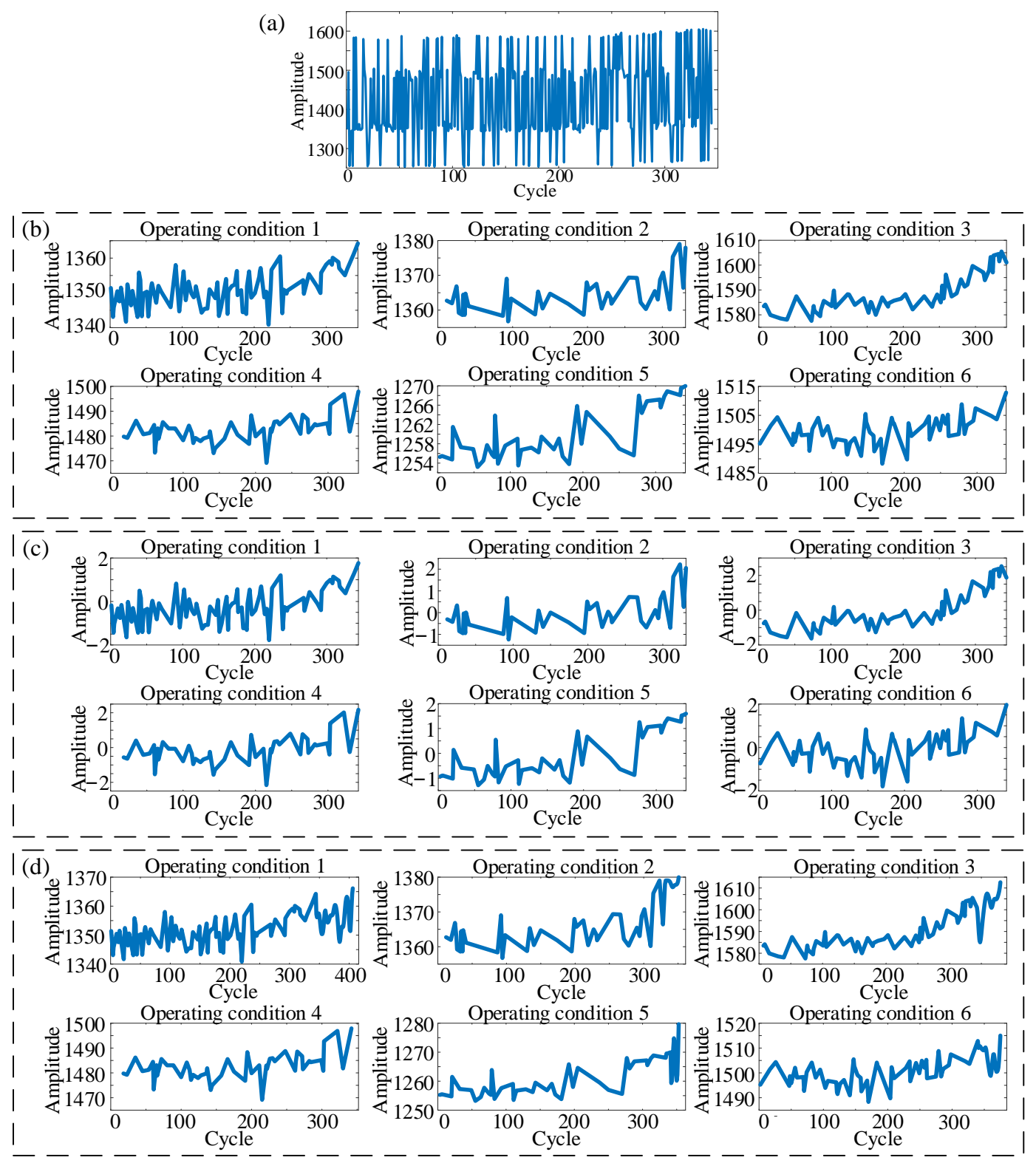

Figure 7. The construction process of the reference degradation model. (a) One of the similarity reference samples, which is selected from the historical sample by measuring the similarity between the sample to be predicted and the historical sample. (b) The operating condition partition of the similarity reference sample. (c) The standardization of operating conditions. (d) The predicted degradation trend of each constant operating condition by RVM.

As the description in Section 4.1, the simulation data have six operating conditions. Therefore, partition the similarity reference sample shown in Figure 7a into six parts according to the 3 operating parameters. The partitioned results are shown in Figure $7 \mathrm{~b}$. Then, as shown in Figure $7 \mathrm{c}$, the standardized samples of the partitioned operating condition are obtained according to Equation (1). Extract the maximum value of each standardized sample as listed in Table 1. Select the maximum of the maximum values of each operating condition and calculate the threshold of each operating condition according to Equation (3). The thresholds are listed in Table 1. The reason for this treatment is that the 
original operating conditions are at different levels, while the standardized operating conditions are at the same level and have a uniform end point of life, as shown in Figure $5 \mathrm{c}$. Therefore, the maximum of the standardized operating conditions is chosen as the end point of life to calculate the life threshold of each operating condition, respectively. The following step is that each operating condition is predicted by RVM according to the life threshold of each operating condition, as shown in Figure 7d. Finally, the reference degradation model is constructed based on the predicted degradation trend and lifecycle. The remaining two similar reference samples are also processed in the same way to construct the degradation models.

Table 1. The maximum value and threshold of each operating condition.

\begin{tabular}{ccccccc}
\hline Operating Condition & $\mathbf{1}$ & $\mathbf{2}$ & $\mathbf{3}$ & $\mathbf{4}$ & $\mathbf{5}$ & $\mathbf{6}$ \\
\hline The maximum value & 2.7825 & 2.8591 & 2.2072 & 2.9129 & 1.7876 & 2.6509 \\
The threshold & 1364.9 & 1379.3 & 1610.8 & 1497.9 & 1276.1 & 1514.2 \\
\hline
\end{tabular}

\subsubsection{The Life Prediction According to Specific Task Requirement}

First, consistent with the operating condition partition of similarity reference samples, the sample to be predicted (as shown in Figure $6 \mathrm{~b}$ ) is partitioned into six parts according to the 3 operating parameters. For each constant operating condition of the sample to be predicted, the life candidate sets and weights under corresponding operating condition are obtained by STM according to the corresponding operating condition in reference degradation models. For the sample shown in Figure $6 \mathrm{~b}$, the weights of each operating condition are shown in Table 2. Since the initial state of the data in set \#2 is different, the life candidate sets are the difference between the initial point of similarity and the lifecycle of corresponding operating condition of historical sample. The initial points and lifecycle of the similarity reference samples are shown in Table 3 and the life candidate sets of each operating condition are shown in Table 4. Then, according to the weights and life candidate sets, the life of each operating condition can be obtained by Equation (4) and the results are listed in Table 5 .

Table 2. The weights of each operating condition.

\begin{tabular}{cccc}
\hline \multirow{2}{*}{ Operating Condition } & \multicolumn{3}{c}{ Similarity Reference Sample } \\
\cline { 2 - 4 } & $\mathbf{1}$ & $\mathbf{2}$ & $\mathbf{3}$ \\
\hline 1 & 0.0715 & 0.0009 & 0.9276 \\
2 & 0.1034 & 0.0009 & 0.8957 \\
3 & 0.8462 & 0.0713 & 0.0825 \\
4 & 0.5876 & 0.1350 & 0.2774 \\
5 & 0.0056 & 0.9910 & 0.0034 \\
6 & 0.7695 & 0.0312 & 0.1993 \\
\hline
\end{tabular}

Table 3. The life information of similarity reference sample.

\begin{tabular}{ccccccc}
\hline & \multicolumn{5}{c}{ Similarity Reference Sample } \\
\cline { 2 - 7 } Operating Condition & \multicolumn{2}{c}{$\mathbf{1}$} & \multicolumn{2}{c}{$\mathbf{2}$} & \multicolumn{3}{c}{$\mathbf{3}$} \\
\cline { 2 - 7 } & Initial Point & Lifecycle & Initial Point & Lifecycle & Initial Point & Lifecycle \\
\hline 1 & 14 & 406 & 3 & 362 & 31 & 334 \\
2 & 7 & 351 & 18 & 339 & 12 & 356 \\
3 & 5 & 380 & 4 & 322 & 10 & 353 \\
4 & 6 & 342 & 18 & 310 & 8 & 336 \\
5 & 9 & 353 & 13 & 338 & 9 & 364 \\
6 & 9 & 375 & 3 & 358 & 2 & 346 \\
\hline
\end{tabular}


Table 4. The life candidate set of each operating condition.

\begin{tabular}{cccc}
\hline \multirow{2}{*}{ Operating Condition } & \multicolumn{3}{c}{ Life Candidate Set } \\
\cline { 2 - 4 } & $\mathbf{1}$ & $\mathbf{2}$ & $\mathbf{3}$ \\
\hline 1 & 392 & 359 & 303 \\
2 & 344 & 321 & 343 \\
3 & 375 & 318 & 343 \\
4 & 336 & 292 & 328 \\
5 & 344 & 325 & 355 \\
6 & 366 & 355 & 344 \\
\hline
\end{tabular}

Table 5. The predicted life of each operating condition by similarity trajectory method (STM).

\begin{tabular}{ccccccc}
\hline Operating Condition & $\mathbf{1}$ & $\mathbf{2}$ & $\mathbf{3}$ & $\mathbf{4}$ & $\mathbf{5}$ & $\mathbf{6}$ \\
\hline Predicted life & 309.4139 & 343.0836 & 368.2959 & 327.8408 & 325.2084 & 361.2722 \\
\hline
\end{tabular}

For the sample to be predicted, as shown in Figure $6 \mathrm{~b}$, the mission profile in the prediction is assumed to be that experienced by the latter $1 / 3$ data. The mission profile of the latter $1 / 3$ data (shown in Figure $6 \mathrm{c}$ ) is shown in Figure 8 according to the 3 operating parameters, and the proportions of each operating condition in the mission profile are listed in Table 6. According to Equation (5), the life of the sample to be predicted under the mission profile experienced by the latter $1 / 3$ data is 334.1180 cycle, which is very close to the actual life of the sample to be predicted.

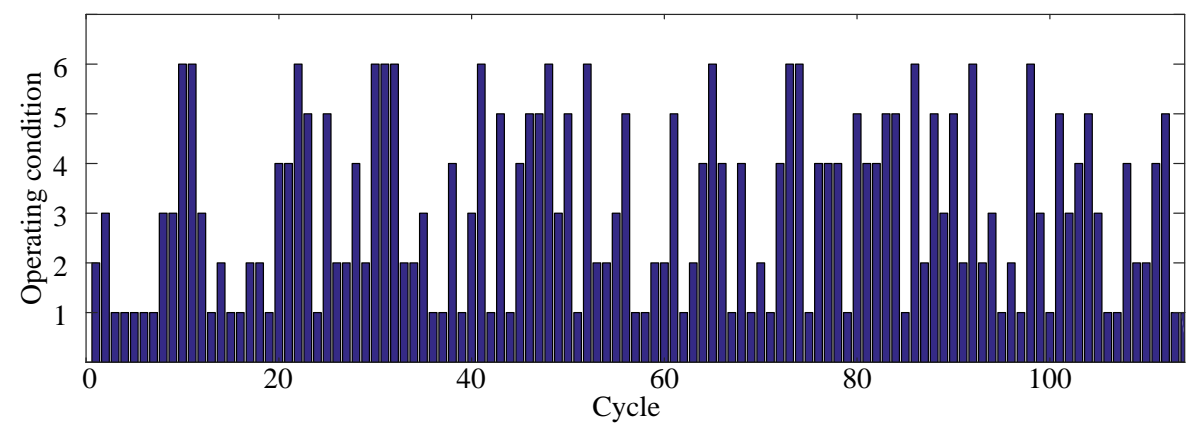

Figure 8. The operating condition of the mission profile experienced by the latter $1 / 3$ data.

Table 6. The proportion of each operating condition under the mission profile experienced by the latter $1 / 3$ data.

\begin{tabular}{ccccccc}
\hline Operating Condition & $\mathbf{1}$ & $\mathbf{2}$ & $\mathbf{3}$ & $\mathbf{4}$ & $\mathbf{5}$ & $\mathbf{6}$ \\
\hline The proportion & $28.07 \%$ & $18.42 \%$ & $11.40 \%$ & $14.91 \%$ & $14.04 \%$ & $13.16 \%$ \\
\hline
\end{tabular}

In addition, the proposed method not only can obtain the prediction results under the above mission profile, but can also obtain the corresponding results even if the mission profile is switched during the prediction process. According to the predicted life of each operating condition and the proportions of the operating conditions in the mission profile, the corresponding results can be obtained by Equation (5). As shown in Table 7, the sample to be predicted (shown in Figure 6b) is assumed to be in the following 3 mission profiles in the prediction. The information of the mission profile is listed in Table 7, and the predicted result under the corresponding mission profile is also listed in Table 7. Therefore, the proposed method can deal well with the problem of mission profile switching in the prediction. 
Table 7. The predicted result under different mission profiles when mission profile switching.

\begin{tabular}{cccc}
\hline Mission Profile & Operating Condition & The Proportion & The Predicted Result \\
\hline & 1 & $3.68 \%$ & \\
2 & $14.47 \%$ & 351.9989 \\
3 & $32.05 \%$ & \\
4 & $5.26 \%$ & 327.6221 \\
& 5 & $14.49 \%$ & \\
& 6 & $30.05 \%$ & \\
& 1 & $41.23 \%$ & \\
2 & 3 & $10.11 \%$ & \\
& 4 & $9.98 \%$ & \\
& 5 & $10.21 \%$ & \\
& 6 & $21.40 \%$ & \\
& 1 & $7.07 \%$ & \\
& 2 & $18.79 \%$ & \\
& 3 & $12.03 \%$ & \\
& 4 & $12.59 \%$ & \\
& 5 & $22.53 \%$ & \\
& 6 & $15.04 \%$ & \\
\hline
\end{tabular}

Through the demonstration of the above specific case, the proposed method not only can obtain the predicted result which is close to the actual life, but can also deal with the problem of mission profile switching.

\subsection{The Prediction Accuracy of the Proposed Method}

Through the detailed description in the above section, it can be seen that the proposed method can provide effective prediction for mission profile switching, where the mission profile contains multiple operating conditions. Meanwhile, the predicted result is close to the actual life. Next, the prediction accuracy of the proposed method will be further validated in this section, where the mission profile is that experienced by the remaining data in the verification process.

\subsubsection{The Prediction Accuracy of Single Sample}

On the basis of the prediction in Section 4.2, the predicted life under different lengths of the sample to be predicted is calculated according to the above steps. The selected lifecycle data are divided into two parts: The sample to be predicted with different lengths and the remaining data, the operating conditions of which are used as the mission profile in the prediction. The predicted results are shown in Figure 9, where the upper and lower limit of the confidence interval is $20 \%$ of the actual RUL.

As shown in Figure 9, the predicted RUL is basically within the confidence interval. Besides, in most cases, the predicted RULs are close to the actual RULs. Therefore, for selected life-cycle data, the proposed method can obtain good prediction results under the mission profile experienced by the remaining data.

\subsubsection{The Prediction Accuracy of Multiple Samples}

For a single lifecycle sample, the proposed method can obtain good prediction results under different lengths. In this section, the applicability of the proposed method will be further verified. Randomly select 100 sets of lifecycle data from set $\# 2$ and calculate the predicted life according to the steps in Section 4.2. The selected lifecycle data are divided into two parts: The first $2 / 3$ is used as the sample to be predicted and the latter $1 / 3$ is used to validate the prediction result under the mission profile experienced by the latter $1 / 3$ data. The results are shown in Figure 10. 


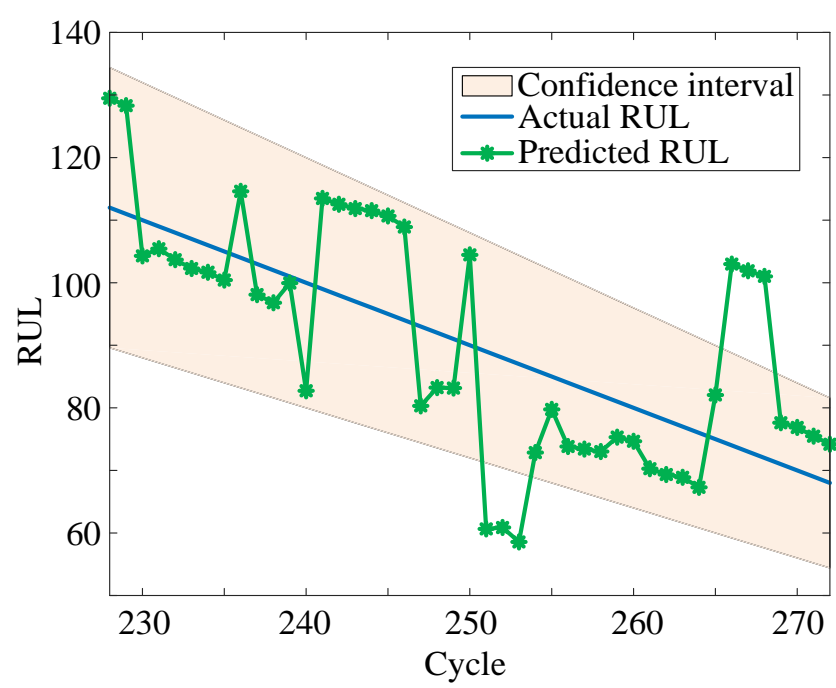

Figure 9. The predicted results under different lengths of the sample to be predicted. RUL, remaining useful life.

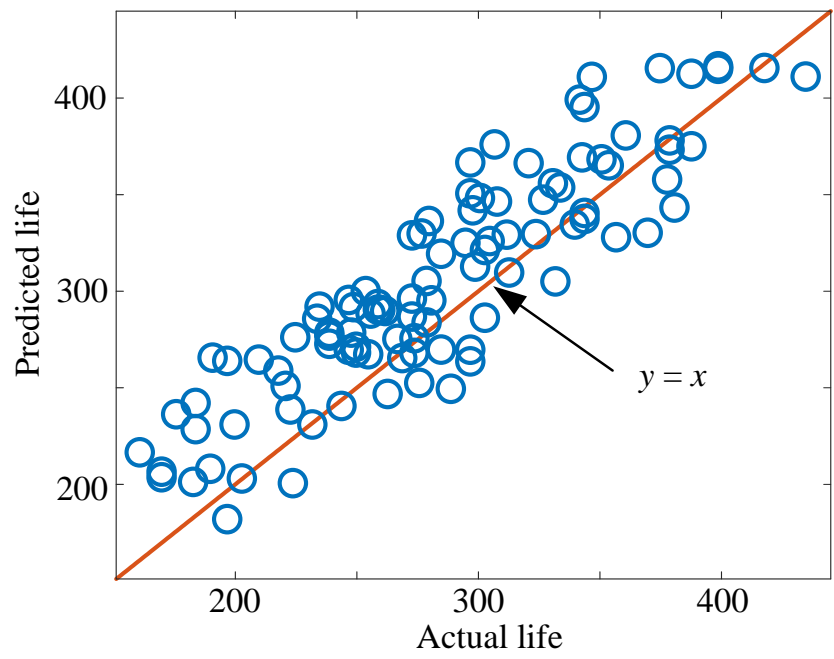

Figure 10. The predicted results of multiple samples.

As shown in Figure 10, the relationship between the predicted life and actual life is approximately linear. The points in Figure 10 fluctuate basically up and down on $y=x$. This means that the predicted life is very close to the actual life. However, most of the points are concentrated on the upper side of the $y=x$. This means the predicted life is larger than the actual life in most cases. Altogether, although the predicted results of the proposed method are larger than the actual results in most cases, the predicted results are very close to the actual results. Therefore, for most lifecycle data, the proposed method can obtain good prediction results when the mission profile is known.

In addition, in the case of multiple samples, we further explain the prediction results of the proposed method under the different lengths of the samples to be predicted and the different number of historical samples simply. Firstly, five samples are selected and, then, for each selected sample, the first $1 / 2,2 / 3$, and $4 / 5$ are used as the samples to be predicted, respectively. Correspondingly, the latter $1 / 2,1 / 3$, and $1 / 5$ are used to verify the RUL prediction results. Secondly, the prediction experiments with 50,100,150, and 200 historical samples are conducted to discuss the influence of the number of historical samples. In addition, in order to eliminate the random influence, 20 trails with randomly 
choosing historical samples under the fixed sample number are employed, and the average Mean Squared Errors (MSEs) are shown in Figure 11, where MSE is calculated as follows:

$$
\text { MSE }=\frac{1}{S N} \sum_{i=1}^{S N}\left(p x_{i}-\hat{x}_{i}\right)^{2}
$$

where $p x_{i}$ is the prediction value of the data set $i, \hat{x}_{i}$ is the real value of the data set $i$, and $S N$ is the number of the predicted data sets.

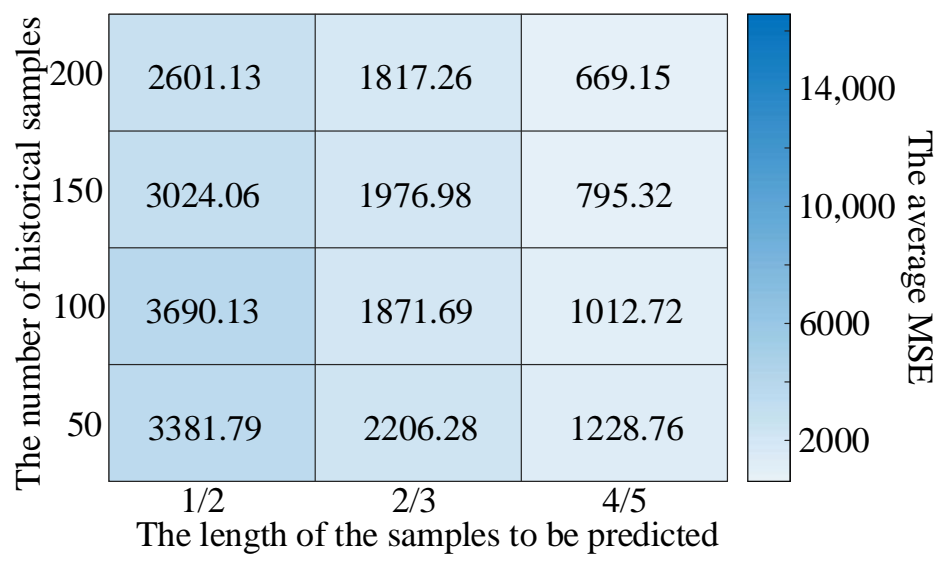

Figure 11. The average Mean Squared Error (MSE) of the proposed method.

As shown in Figure 11, the MSE of the proposed method decreases with the increasing length of the samples to be predicted. Meanwhile, the MSE of the proposed method decreases with the increasing number of historical samples. These phenomena are basically consistent with common sense. Meanwhile, the focus of our proposed method is to solve the problem that most of the current prediction methods cannot solve, rather than to improve the prediction accuracy of the conventional problems. Therefore, we did not discuss the accuracy of the prediction results in detail in the manuscript so as not to affect the focus of our discussion.

In summary, the proposed method can obtain excellent predicted results with the given mission profile. Furthermore, even if the mission profile is switched during the prediction process, the proposed method can obtain corresponding predicted results according to the switched mission profile.

\section{Conclusions}

This paper proposes a new life prediction scheme which can predict the life when considering the mission profile switching. First of all, the reference degradation models are constructed according to the RVM prediction results of each constant operating condition. The RVM achieves a more complete description of the detail and trend information of the degradation curve simultaneously. Therefore, more accurate life prediction results under constant operating conditions are obtained by RVM. Then, the life of the constant operating conditions of the sample to be predicted is obtained by STM. Finally, according to the proportion of each constant operating condition in the mission profile, the final life is calculated through weighted aggregating the life of each constant operating condition of the sample to be predicted. Through the experiments organized using the NASA turbofan engine simulation dataset, it is proved that the proposed method has excellent predicted results with the given mission profile. In addition, the proposed method can obtain the corresponding life predicted results according to mission profile switching.

Although some important techniques associated with the proposed method have been investigated in this paper, there are still a few questions that are worthy of further consideration. First, the similarity measurement method based on Euclidean distance is the simplest but not the best one. Second, switching 
of the mission profile with the same proportion of operating conditions is not considered in this paper. Therefore, the proposed method may be further improved by considering the above questions.

Author Contributions: Conceptualization, J.Y.; formal analysis, J.Y.; investigation, J.Y., H.Z., and Y.Y.; methodology, J.Y.; project administration, M.X.; software, J.Y. and Y.Y.; supervision, M.X.; validation, H.Z.; writing-original draft, J.Y.; writing - review and editing, J.Y., H.Z., Y.Y., and M.X. All authors have read and agreed to the published version of the manuscript.

Funding: This research was funded by the National Natural Science Foundation of China, grant number 11172078.

Conflicts of Interest: The authors declare no conflicts of interest.

\section{References}

1. Lee, J.; Wu, F.; Zhao, W.; Ghaffari, M.; Liao, L.; Siegel, D. Prognostics and health management design for rotary machinery systems-Reviews, methodology and applications. Mech. Syst. Signal Process. 2014, 42, 314-334. [CrossRef]

2. Lei, Y.; Li, N.; Guo, L.; Li, N.; Yan, T.; Lin, J. Machinery health prognostics: A systematic review from data acquisition to RUL prediction. Mech. Syst. Signal Process. 2018, 104, 799-834. [CrossRef]

3. Li, Y.; Billington, S.; Zhang, C.; Kurfess, T.; Danyluk, S.; Liang, S. Adaptive prognostics for rolling element bearing condition. Mech. Syst. Signal Process. 1999, 13, 103-113. [CrossRef]

4. Li, C.J.; Lee, H. Gear fatigue crack prognosis using embedded model, gear dynamic model and fracture mechanics. Mech. Syst. Signal Process. 2005, 19, 836-846. [CrossRef]

5. Oppenheimer, C.H.; Loparo, K.A. Physically based diagnosis and prognosis of cracked rotor shafts. In Proceedings of the 2nd Conference on Component and Systems Diagnostics, Prognostics, and Health Management, Orlando, FL, USA, 1-5 April 2002; pp. 122-132. [CrossRef]

6. Guo, L.; Li, N.; Jia, F.; Lei, Y.; Lin, J. A recurrent neural network based health indicator for remaining useful life prediction of bearings. Neurocomputing 2017, 240, 98-109. [CrossRef]

7. Zhu, J.; Chen, N.; Peng, W. Estimation of Bearing Remaining Useful Life based on Multiscale Convolutional Neural Network. IEEE Trans. Ind. Electron. 2018, 66, 3208-3216. [CrossRef]

8. Ren, L.; Sun, Y.; Cui, J.; Zhang, L. Bearing remaining useful life prediction based on deep autoencoder and deep neural networks. J. Manuf. Syst. 2018, 48, 71-77. [CrossRef]

9. Hinchi, A.Z.; Tkiouat, M. Rolling element bearing remaining useful life estimation based on a convolutional long-short-term memory network. Procedia Comput. Sci. 2018, 127, 123-132. [CrossRef]

10. Zhang, Y.; Xiong, R.; He, H.; Pecht, M. Long short-term memory recurrent neural network for remaining useful life prediction of lithium-ion batteries. IEEE Trans. Veh. Technol. 2018, 67, 5695-5705. [CrossRef]

11. Wu, Y.; Wang, Y.; Jiang, Y.; Quan, S. A Prognostic method for DC-DC converters under variable operating conditions. In Proceedings of the 2014 International Conference on Prognostics and Health Management, Cheney, WA, USA, 22-25 June 2014. [CrossRef]

12. Jiang, Y.; Wang, Y.; Quan, S.; Yi, W. A fault prediction method for closed-loop SEPIC converters under variable operating conditions. In Proceedings of the 2016 Prognostics and System Health Management Conference, Chengdu, China, 19-21 October 2016. [CrossRef]

13. Rigamonti, M.; Baraldi, P.; Zio, E.; Astigarraga, D.; Galarza, A. Particle Filter-Based Prognostics for an Electrolytic Capacitor Working in Variable Operating Conditions. IEEE Trans. Power Electron. 2015, 31, 1567-1575. [CrossRef]

14. Yan, H.; Liu, K.; Xi, Z.; Shi, J. Multiple Sensor Data Fusion for Degradation Modeling and Prognostics under Multiple Operational Conditions. IEEE Trans. Reliab. 2016, 65, 1416-1426. [CrossRef]

15. Sameer, A.; Maio, F.D.; Baraldi, P.; Zio, E. Remaining useful life estimation in heterogeneous fleets working under variable operating conditions. Reliab. Eng. Syst. Saf. 2016, 156, 109-124. [CrossRef]

16. Tao, T.; Zio, E.; Wei, Z. A novel support vector regression method for online reliability prediction under multi-state varying operating conditions. Reliab. Eng. Syst. Saf. 2018, 177, 35-49. [CrossRef]

17. You, M.Y.; Meng, G. A Framework of Similarity-Based Residual Life Prediction Approaches Using Degradation Histories with Failure, Preventive Maintenance, and Suspension Events. IEEE Trans. Reliab. 2012, 62, 127-135. [CrossRef] 
18. Zhang, Q.; Tse, W.T.; Wan, X.; Xu, G. Remaining useful life estimation for mechanical systems based on similarity of phase space trajectory. Expert Syst. Appl. 2015, 42, 2353-2360. [CrossRef]

19. You, M.Y.; Meng, G. Toward effective utilization of similarity based residual life prediction methods: Weight allocation, prediction robustness, and prediction uncertainty. Proc. Inst. Mech. Eng. Part E J. Process Mech. Eng. 2013, 227, 74-84. [CrossRef]

20. Wang, T.; Yu, J.; Siegel, D.; Lee, J. A similarity-based prognostics approach for Remaining Useful Life estimation of engineered systems. In Proceedings of the International Conference on Prognostics and Health Management, Denver, CO, USA, 6-9 October 2008; pp. 1-6. [CrossRef]

21. Zio, E.; Di Maio, F. A data-driven fuzzy approach for predicting the remaining useful life in dynamic failure scenarios of a nuclear system. Reliab. Eng. Syst. Saf. 2010, 95, 49-57. [CrossRef]

22. Wang, P.; Youn, B.D.; Hu, C. A generic probabilistic framework for structural health prognostics and uncertainty management. Mech. Syst. Signal Process. 2012, 28, 622-637. [CrossRef]

23. Li, L.L.; Ma, D.J.; Li, Z.G. Residual Useful Life Estimation by a Data-Driven Similarity-Based Approach: A Data-Driven Similarity-Based Approach. Qual. Reliab. Eng. Int. 2016, 33, 231-239. [CrossRef]

24. Khelif, R.; Malinowski, S.; Chebel-Morello, B.; Zerhouni, N. RUL prediction based on a new similarity-instance based approach. In Proceedings of the IEEE 23rd International Symposium on Industrial Electronics, Istanbul, Turkey, 1-4 June 2014; pp. 2463-2468. [CrossRef]

25. Liang, Z.; Gao, J.; Jiang, H.; Gao, X.; Gao, Z.; Wang, R. A similarity-based method for remaining useful life prediction based on operational reliability. Appl. Intell. 2018, 48, 2983-2995. [CrossRef]

26. Liang, Z.; Gao, J.; Jiang, H.; Gao, X.; Gao, Z.; Wang, R. A Degradation Degree Considered Method for Remaining Useful Life Prediction Based on Similarity. Comput. Sci. Eng. 2018, 21, 50-64. [CrossRef]

27. Liao, H.; Tian, Z. A framework for predicting the remaining useful life of a single unit under time-varying operating conditions. IIE Trans. 2013, 45, 964-980. [CrossRef]

28. Si, X.S.; Hu, C.H.; Kong, X.; Zhou, D.H. A Residual Storage Life Prediction Approach for Systems with Operation State Switches. IEEE Trans. Ind. Electron. 2014, 61, 6304-6315. [CrossRef]

29. Liu, T.; Quan, S.; Jing, F.; Pan, Z.; Huangpeng, Q. Residual life estimation under time-varying conditions based on a Wiener process. J. Stat. Comput. Simul. 2017, 87, 211-226. [CrossRef]

30. Gebraeel, N.; Pan, J. Prognostic Degradation Models for Computing and Updating Residual Life Distributions in a Time-Varying Environment. IEEE Trans. Reliab. 2008, 57, 539-550. [CrossRef]

31. Zhao, F.; Tian, Z.; Bechhoefer, E.; Yong, Z. An Integrated Prognostics Method under Time-Varying Operating Conditions. IEEE Trans. Reliab. 2015, 64, 673-686. [CrossRef]

32. Zhang, Z.X.; Si, X.S.; Hu, C.H.; Pecht, M.G. A Prognostic Model for Stochastic Degrading Systems with State Recovery: Application to Li-Ion Batteries. IEEE Trans. Reliab. 2017, 66, 1293-1308. [CrossRef]

33. U. S. Military Handbook 338: Electronic Reliability Design Handbook; US Dept. Def.: Arlington County, VA, USA, 1998; chapter 6; pp. 1-74.

34. Caesarendra, W.; Widodo, A.; Yang, B.S. Application of relevance vector machine and logistic regression for machine degradation assessment. Mech. Syst. Signal Process. 2010, 24, 1161-1171. [CrossRef]

35. Widodo, A.; Yang, B.S. Application of relevance vector machine and survival probability to machine degradation assessment. Expert Syst. Appl. 2011, 38, 2592-2599. [CrossRef]

36. Turbofan Engine Degradation Simulation Data Set. Available online: https:/ti.arc.nasa.gov/m/project/ prognostic-repository/CMAPSSData.zip (accessed on 20 November 2017).

37. Saxena, A.; Kai, G.; Simon, D.; Eklund, N. Damage propagation modeling for aircraft engine run-to-failure simulation. In Proceedings of the International Conference on Prognostics and Health Management, Denver, CO, USA, 6-9 October 2008; pp. 1-9. [CrossRef]

38. Xin, Z.; Xiao, M.Q.; Xie, Y.W.L.; Huang, H.Q.; Wei, C. A method for predicting aviation equipment failures based on degradation-track similarity. In Proceedings of the IEEE Chinese Guidance, Navigation and Control Conference, Nanjing, China, 12-14 August 2016; pp. 1472-1477. [CrossRef]

(C) 2020 by the authors. Licensee MDPI, Basel, Switzerland. This article is an open access article distributed under the terms and conditions of the Creative Commons Attribution (CC BY) license (http://creativecommons.org/licenses/by/4.0/). 